\title{
Research Article \\ Effect of Strain on Thermal Conductivity of Si Thin Films
}

\author{
Xingli Zhang' and Guoqiang $\mathrm{Wu}^{2}$ \\ ${ }^{1}$ College of Mechanical and Electrical Engineering, Northeast Forestry University, Harbin 150040, China \\ ${ }^{2}$ The State Key Laboratory of Structural Analysis for Industrial Equipment, Dalian University of Technology, Dalian 116024, China
}

Correspondence should be addressed to Xingli Zhang; zhang-xingli@nefu.edu.cn

Received 1 March 2016; Revised 29 April 2016; Accepted 10 May 2016

Academic Editor: Yan Wang

Copyright (C) 2016 X. Zhang and G. Wu. This is an open access article distributed under the Creative Commons Attribution License, which permits unrestricted use, distribution, and reproduction in any medium, provided the original work is properly cited.

\begin{abstract}
Nonequilibrium molecular dynamics (NEMD) simulations are employed to gain an understanding of the effect of strain on the thermal conductivity of Si thin films. The analysis shows that the strain has an appreciable influence on the thermal conductivity of Si thin films. The thermal conductivity decreases as the tensile strain increases and increases as the compressive strain increases. The decrease of the phonon velocities and surface reconstructions generated under strain could explain well the effects of strain on the thermal conductivity of Si thin films.
\end{abstract}

\section{Introduction}

The Si thin films have shown important applications in many fields, such as microelectronic, optoelectronic, and microelectromechanical devices. Understanding and predicting thermal transport of Si thin films at the nanoscale are essential for improving the performance and reliability of these devices. Because the characterized size is down to the nanoscale, the heat transfer of $\mathrm{Si}$ thin films becomes unusual as compared to bulk materials. Researchers can design nanomaterials with certain thermal properties through probing the factors which affect thermal properties, so the thermal conductivity of Si thin films depending on the size of the system and defects have been researched by Boltzmann transport equation (BTE) based approaches and molecular dynamics (MD) simulations [1-5].

Nanoscale devices, particularly thin films, usually contain residual strain after fabrication [6], which may considerably affect the thermal transport properties of the material. Many researchers have attempted to simulate the effect of strain on the thermal properties of material using different methods. Bhowmick and Shenoy [7] derived the relationship of thermal conductivity as a function of temperature and strain using the Peierls-Boltzmann formulation; Zhang et al. [8] investigated how the thermal conductivity responded to the external tensile strain by nonequilibrium molecular dynamics (NEMD) simulations on functionalized bilayer graphene sheet; Wang and Shen [9] investigated the thermal properties of $\mathrm{Ni} / \mathrm{Al}$ laminated structures using NEMD method; $\mathrm{Xu}$ and $\mathrm{Li}$ [10] presented a model that combined lattice dynamics and the phonon BTE to analyze strain effect on the cross-plane phonon thermal conductivity of silicon wire-germanium host nanocomposites. These studies have shown the significance of strain on the nanoscale thermal transport.

In this work, we carry out NEMD simulations to investigate the thermal properties of Si thin films. We find that strain has a strong influence on the thermal conductivity of thin films. Our work suggests that applying strain could serve as a means of tuning the thermal conductivity of nanoscale material.

\section{Molecular Dynamic Simulation}

The NEMD simulations of the Si thin films are performed using LAMMPS code. Figure 1 shows the simulation model in the present research. At the initialized condition, the positions of each atom are arranged according to the crystal lattice structure, and atomic velocities meet the MaxwellBoltzmann distribution function. The thickness of the $\mathrm{Si}$ thin films chosen for this study is $10 \AA, 15 \AA, 20 \AA$, and $50 \AA$ respectively, where $\AA$ is the lattice constant of $S i$. The periodic boundary conditions are applied in $X$ and $Z$ direction, and the free boundary conditions are applied in $Y$ direction. 


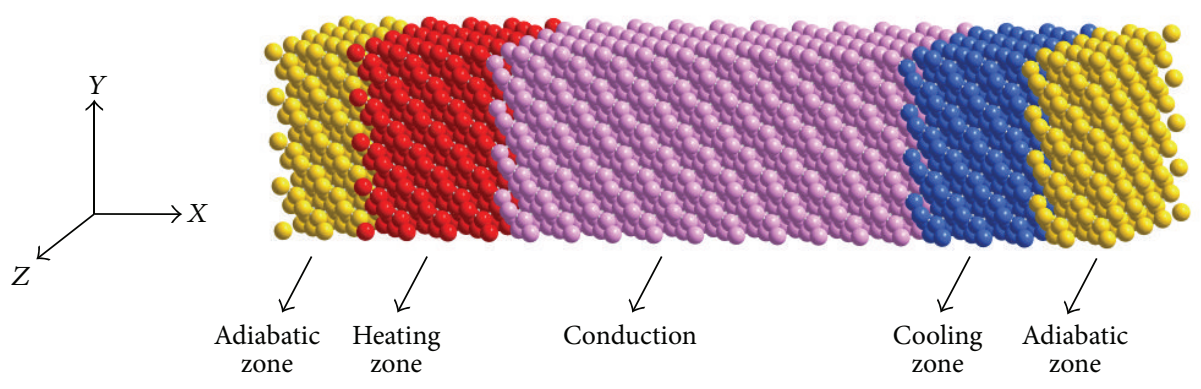

FIGURE 1: MD simulation model in Si thin film.

The cross-sectional area of $Y O Z$ is $4 \AA \times 4 \AA$. The heating and cooling zones with the thickness of $3 \AA$ create a temperature gradient in the system by controlling the energy given or taken from these regions. Adiabatic zones with the thickness of $2 \AA$ are constructed to prevent the atoms escaping from the system, and the velocities of each atom in this region are 0.

Tersoff three-body potential model is employed to describe the interaction among different $\mathrm{Si}$ atoms in the simulation film that is reliable to describe the semiconductor atoms potential properties [11]. The Newton's classical equations of motion for those atoms are solved with the VelocityVerlet integration algorithm. The time step for the simulation is set as $1 \mathrm{fs}$. The simulations consist of two stages: the first stage is the constant-temperature simulation, in which the temperature is maintained at constant value with a coupling time of $2 \times 10^{6}$ MD steps; the second stage is the constantenergy one with a coupling time of $8 \times 10^{6} \mathrm{MD}$ steps to ensure that the whole system reached an equilibrium state. The method proposed by Juncl and Jullien [12] is used to apply a specified heat flux by scaling the velocities of the atoms in the heating and cooling zones of the simulations. A specified amount of energy $\Delta E$ (set to $1 \%$ of $k_{B} T$ ) is added to the heating zone and the same amount of energy is subtracted from the cooling zone at each time step.

The velocities of all the particles in the heating and cooling zones are scaled according to the following equation:

$$
v_{i, \text { new }}=v_{G}+\alpha\left(v_{i, \text { old }}-v_{G}\right),
$$

where $v_{G}$ is the velocity of the center mass, which is set as zero at the beginning of our simulations. A scaling factor $\alpha$ is calculated from the following equation:

$$
\alpha=\sqrt{1 \pm \frac{\Delta E}{\Delta E_{k}}}
$$

where $E_{k}=\sum_{i=1}^{N}(1 / 2) m_{i} v_{i}^{2}$ is the kinetic energy of atoms in the hot or cold reservoirs.

Based on Fourier's law of conduction, the thermal conductivity is given as

$$
\lambda=\frac{\Delta E}{2 A \tau \cdot(\partial T / \partial x)},
$$

where $\tau$ is the simulation step time and $A$ is the crosssectional area. To obtain the temperature distribution,

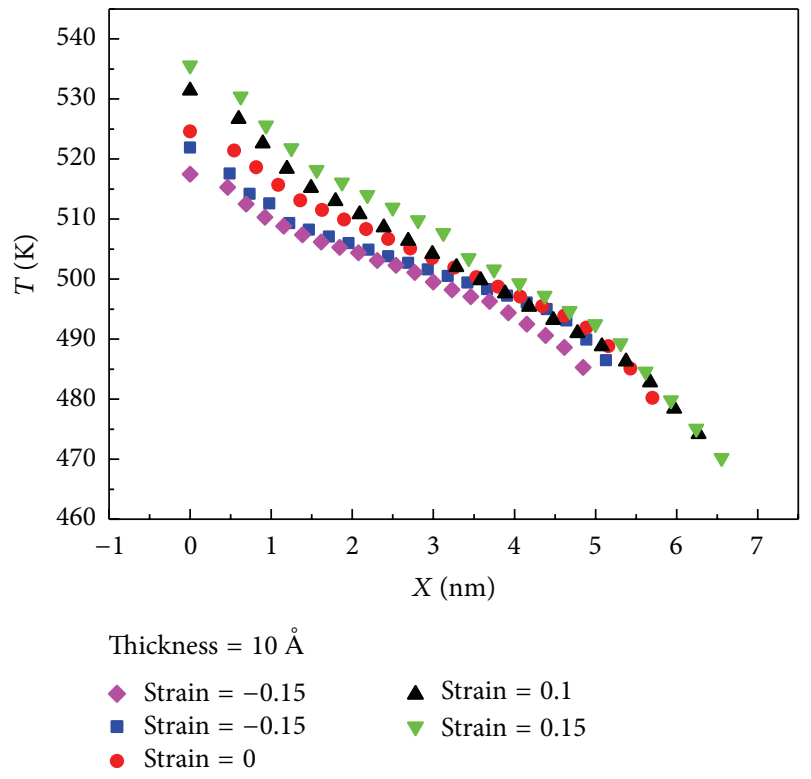

FIGURE 2: Temperature profile in the direction of heat transfer.

the system is divided into 20 planes along the $X$ direction, and the instantaneous temperature of each plane is calculated using the following equation:

$$
T=\frac{1}{3 k_{B} N}\left\langle\sum_{i=1}^{N}\left(\frac{1}{2} m_{i} v_{i}^{2}\right)\right\rangle,
$$

where $m_{i}$ is the mass of atoms, $v_{i}$ is the velocity of atoms, $N$ is the number of atoms in each plane, $k_{B}$ is Boltzmann constant, and $k_{B}=1.38 \times 10^{-23} \mathrm{~J} / \mathrm{K}$.

For the analysis, $0 \sim 15 \%$ tensile and compressive strain is imposed on our NEMD simulation system by recalibrating the atomic coordinates. The rate is $0.005 \mathrm{~nm}$ per $10^{5}$ steps. When each load applying process is finished, the thin films need to achieve equilibrium for a certain relaxation time.

\section{Results and Discussion}

All the simulations are implemented at $500 \mathrm{~K}$. Figure 2 displays the calculated temperature distributions in the film with strain of $-0.1,0$, and 0.1 , respectively. The typical distributions have been obtained in all simulation runs. Both ends of 


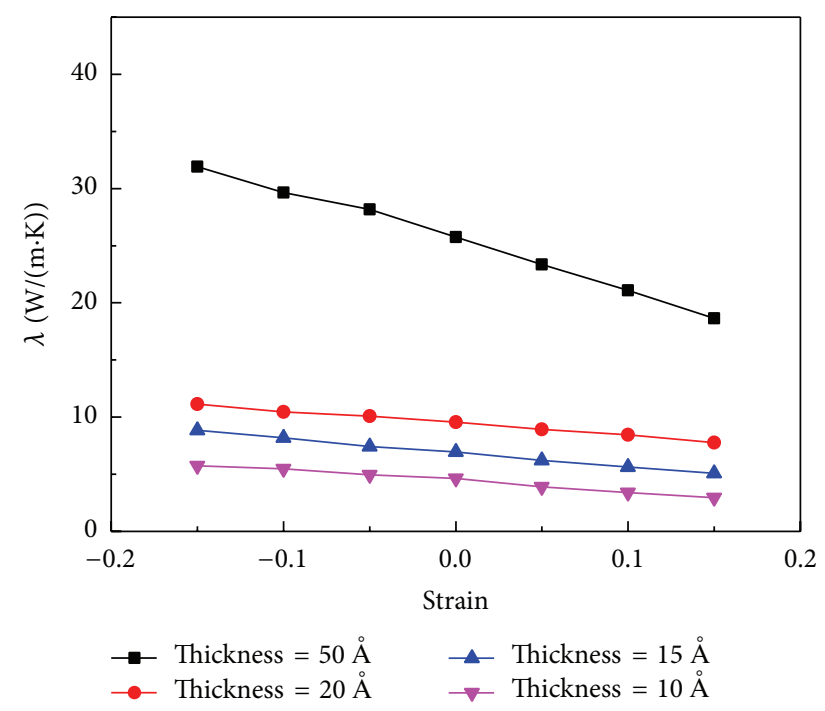

FIGURE 3: Thermal conductivity variation with strain.

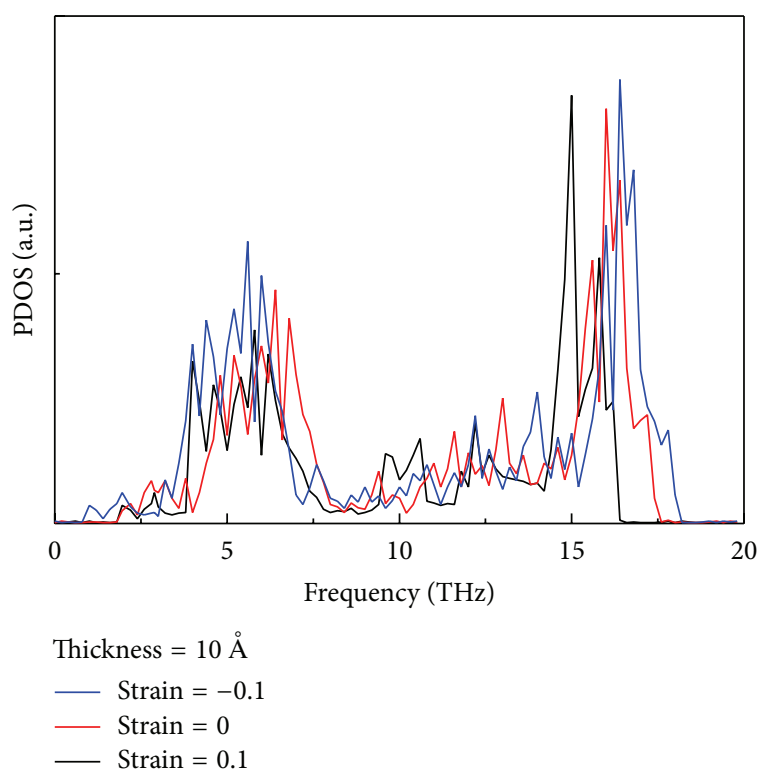

FIGURE 4: Strain dependent phonon density of states of Si thin films.

into low frequency phonons, and this shift could slow down the phonon group velocities [17]. Under compressive strain, most phonons will be at a higher frequency which could enhance thermal transportation capacity [18].

In gas kinetic theory, the lattice thermal conductivity can be calculated using the following expression:

$$
\lambda=\frac{1}{3} C v \Lambda_{\mathrm{eff}},
$$

where $C$ is the heat capacity per unit volume and $v$ and $\Lambda_{\text {eff }}$ are the velocity of sound and mean free path of the phonons, respectively. It can be seen from (5) that the decline of phonon velocities due to the tensile strain will decrease the thermal conductivity of thin films.

On the other hand, the surface reconstructions of the simulation system contribute to the change in thermal conductivity. In Figures 5 and 6, the Si thin films with the thickness of $10 \AA$ under different tensile and compressive strain exhibit different atomic configuration characteristics. Under small strain (Figures 5(a), 5(b), 6(a), and 6(b)), the surface disorder is significantly small, and there is no plastic deformation in the simulation system. As the applied strain is increased, dislocations could be generation in the $\mathrm{Si}$ thin films under large strain due to the fact that the atoms will recombine with each other to form new bonds. Figures 5(c), 5(d), 6(c), and $6(\mathrm{~d})$ show that the disordered region expands to the whole conducting zones and the plastic deformation occurs simulations result that the partial dislocations are nucleated raising the contribution of extended full dislocations [19]. The simulation partial dislocations are even separated into two pieces in our simulation with the tensile strain of 0.15 . The surface reconstructions can scatter phonons significantly which leads to a reduction of the thermal conductivity. For the compressive strain increase to 0.15 , a convex distortion is gradually, which are stable agreeing with the dislocation

formed near the center of the thin films, while this structure right when the compressive strain is applied. The results imply that the tensile strain can shift the high frequency phonons

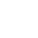

the temperature profile show a nonlinear trend attributed to the strong scattering of heating and cooling zones. A linear emperature profile exists in the middle regions, and the the thermal conductivity is collected in Figure 3. It is noted that the thermal conductivity decreases as the tensile strain the thermal conductivity of the thin films with the thickness therma which may suggest that the strain effect on the configuration over all the sizes. When the thickness of thin film is $10 \AA$, the thermal conductivity from its initial value increases to 0.1 and increases by $18 \%$ as the compressive tran 0.1 . Such trend is consistent with the $[13,14]$. It is also observed from Figure 3 that the thermal inity of $\mathrm{Si}$ thin films increases with the incre experimental and computational works. The explanation of discretisation of the wave vectors for small systems and the

To explain the coupling between strain and thermal quency phonons play a much more important role in thermal conduction of thin films. The peak moves towards the left when the tensile strain is applied, while it moves towards the 


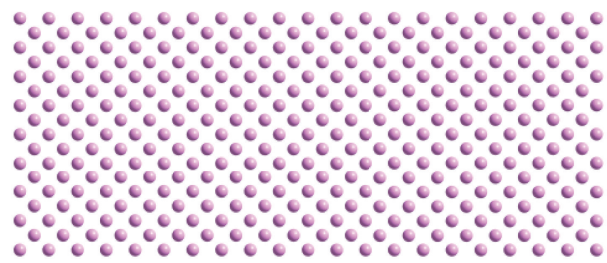

(a) $0 \%$ tensile strain

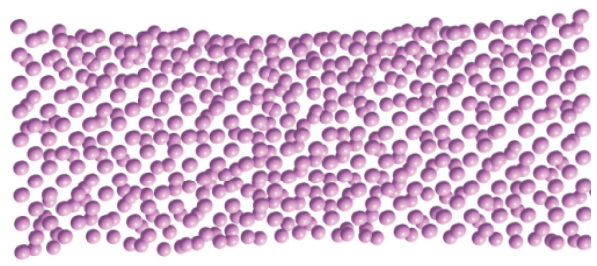

(c) $10 \%$ tensile strain

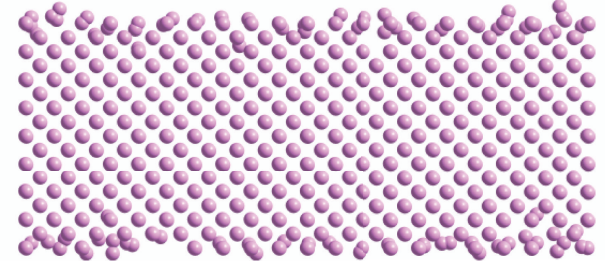

(b) $5 \%$ tensile strain

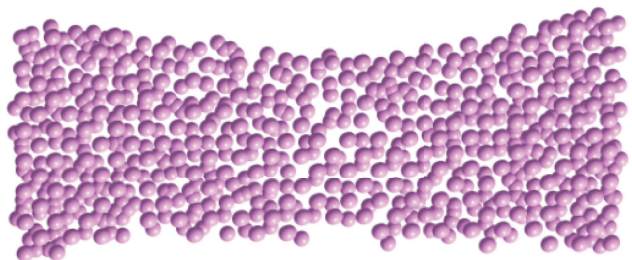

(d) $15 \%$ tensile strain

FIgURE 5: Configuration plots of Si thin films as the tensile strain increases.

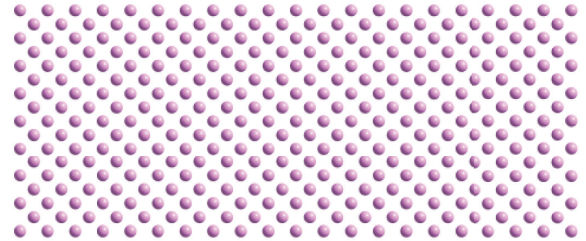

(a) $0 \%$ compressive strain

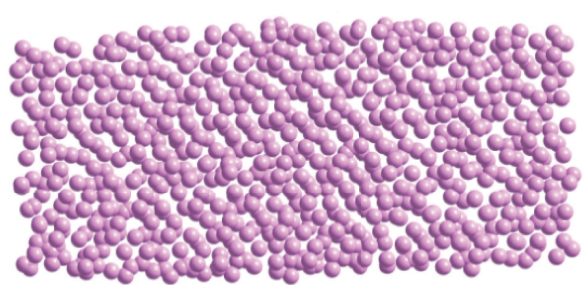

(c) $10 \%$ compressive strain

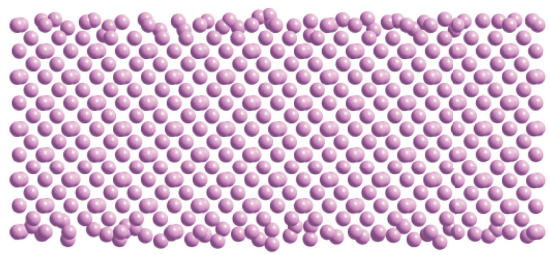

(b) $5 \%$ compressive strain

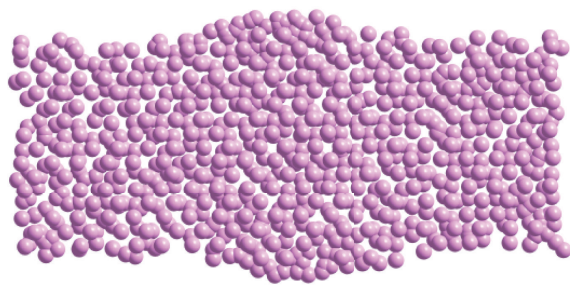

(d) $15 \%$ compressive strain

FIgURE 6: Configuration plots of Si thin films as the compressive strain increases.

transformation does not reduce the thermal conductivity. It may be due to the fact that the surface effect is lower than the effect of phonon group velocity under compressive strain.

\section{Conclusions}

This study has performed an investigation into the thermal conductivities of $\mathrm{Si}$ thin films with varying strain. The simulation used the NEMD method to obtain a temperature gradient, and the thermal conductivities are then evaluated using the heat current and temperature gradient. The results have shown that the thermal conductivity of the Si thin films could be significantly decreased or increased by tensile or compressive strain. This is due to the mode-specific group velocities of phonons decreasing continuously from compressive strain to tensile strain. In addition, the surface disorder is significantly increased from compressive strain to tensile strain. Our demonstration of large changes in thermal conductivity driven by strain may point to a new direction of dynamic thermal management.

\section{Competing Interests}

The authors declare no competing interests.

\section{Acknowledgments}

The project was supported by the Fundamental Research Funds for the Central Universities of China (Grant no. DL12BB38). 


\section{References}

[1] D. Terris, K. Joulain, D. Lemonnier, D. Lacroix, and P. Chantrenne, "Prediction of the thermal conductivity anisotropy of Si nanofilms: results of several numerical methods," International Journal of Thermal Sciences, vol. 48, no. 8, pp. 1467-1476, 2009.

[2] S. Ju and X. Liang, "Thermal conductivity of nanocrystalline silicon by direct molecular dynamics simulation," Journal of Applied Physics, vol. 112, no. 6, article 064305, 2012.

[3] A. Bodapati, P. K. Schelling, S. R. Phillpot, and P. Keblinski, "Vibrations and thermal transport in nanocrystalline silicon," Physical Review B, vol. 74, no. 24, Article ID 245207, 2006.

[4] S. H. Ju, X. G. Liang, and X. H. Xu, "Out-of-plane thermal conductivity of polycrystalline silicon nanofilm by molecular dynamics simulation," Journal of Applied Physics, vol. 110, no. 5, Article ID 054318, 2011.

[5] X. L. Zhang and Z. W. Sun, "Effects of vacancy structural defects on the thermal conductivity of silicon thin films," Semiconductor, vol. 32, Article ID 053002, 2011.

[6] M. Adamcyk, J. H. Schmid, T. Tiedje et al., "Comparison of strain relaxation in InGaAsN and InGaAs thin films," Applied Physics Letters, vol. 80, no. 23, pp. 4357-4359, 2002.

[7] S. Bhowmick and V. B. Shenoy, "Effect of strain on the thermal conductivity of solids," Journal of Chemical Physics, vol. 125, no. 16, article 164513, 2006.

[8] Y. Y. Zhang, Q. X. Pei, X. Q. He, and Y.-W. Mai, "A molecular dynamics simulation study on thermal conductivity of functionalized bilayer graphene sheet," Chemical Physics Letters, vol. 622, pp. 104-108, 2015.

[9] X. Wang and S. P. Shen, "Effects of temperature and strain on thermal properties of Ni/Al laminated structure," Computational Materials Science, vol. 84, pp. 13-17, 2014.

[10] Y. Xu and G. Li, "Strain effect analysis on phonon thermal conductivity of two-dimensional nanocomposites," Journal of Applied Physics, vol. 106, no. 11, Article ID 114302, 2009.

[11] J. Tersoff, "New empirical approach for the structure and energy of covalent systems," Physical Review B, vol. 37, no. 12, pp. 69917000, 1988.

[12] P. Juncl and R. Jullien, "Molecular-dynamics calculation of the thermal conductivity of vitreous silica," Physical Review BCondensed Matter and Materials Physics, vol. 59, no. 21, pp. 13707-13711, 1999.

[13] K. Jung, M. Cho, and M. Zhou, "Thermal and mechanical response of [0001]-oriented $\mathrm{GaN}$ nanowires during tensile loading and unloading," Journal of Applied Physics, vol. 112, no. 8, Article ID 083522, 2012.

[14] X. Li, K. Maute, M. L. Dunn, and R. Yang, "Strain effects on the thermal conductivity of nanostructures," Physical Review B, vol. 81, no. 24, Article ID 245318, 2010.

[15] P. K. Schelling, S. R. Phillpot, and P. Keblinski, "Comparison of atomic-level simulation methods for computing thermal conductivity," Physical Review B-Condensed Matter and Materials Physics, vol. 65, no. 14, Article ID 144306, 2002.

[16] P. Chantrenne and J.-L. Barrat, "Finite size effects in determination of thermal conductivities: comparing molecular dynamics results with simple models," Journal of Heat Transfer, vol. 126, no. 4, pp. 577-585, 2004.

[17] Z. Xu and M. J. Buehler, "Strain controlled thermomutability of single-walled carbon nanotubes," Nanotechnology, vol. 20, no. 18, Article ID 185701, 2009.
[18] J. Zhang, X. He, L. Yang et al., "Effect of tensile strain on thermal conductivity in monolayer graphene nanoribbons: a molecular dynamics study," Sensors, vol. 13, no. 7, pp. 9388-9395, 2013.

[19] L. Cao, A. Hunter, I. J. Beyerlein, and M. Koslowski, “The role of partial mediated slip during quasi-static deformation of 3D nanocrystalline metals," Journal of the Mechanics and Physics of Solids, vol. 78, pp. 415-426, 2015. 

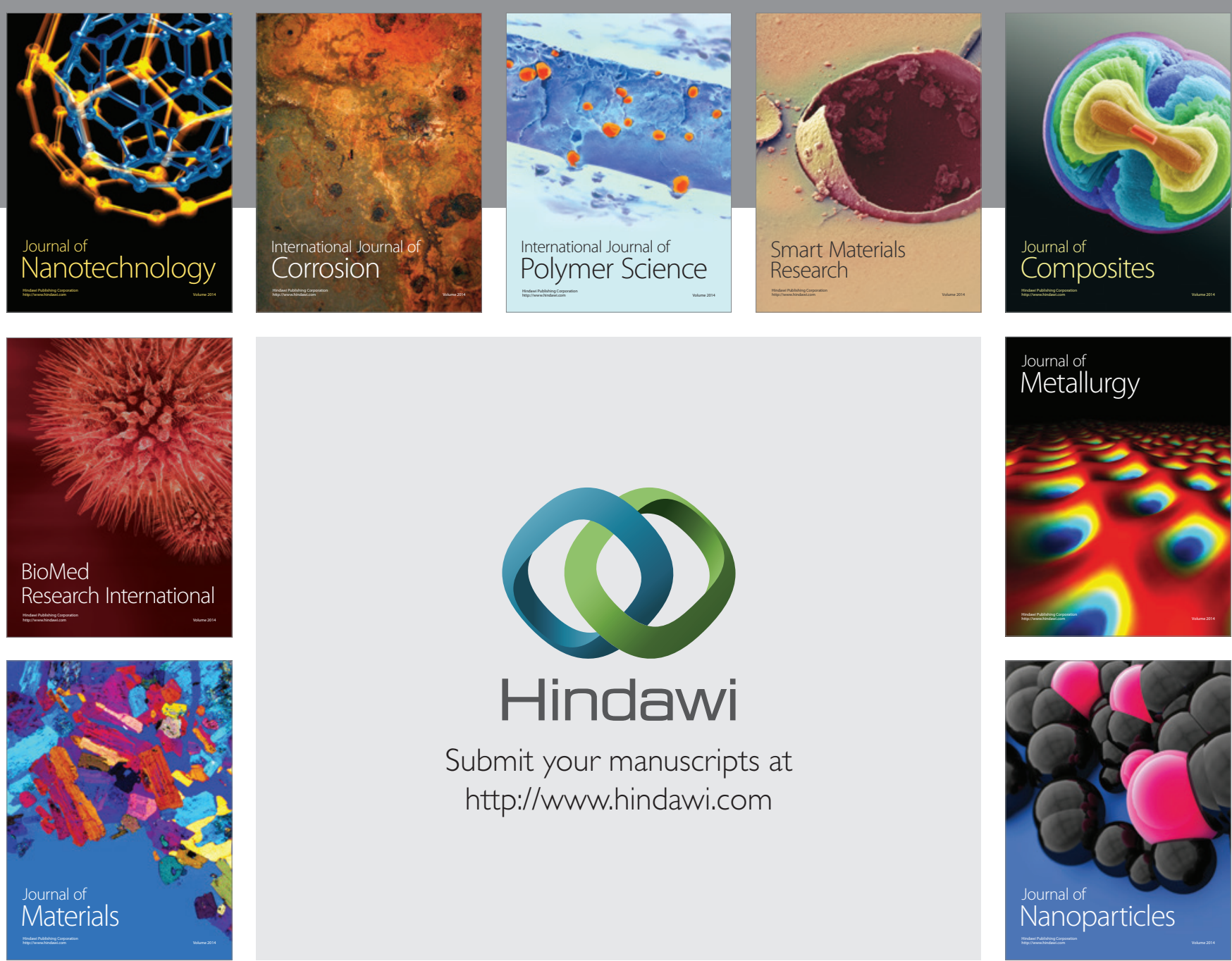

\section{Hindawi}

Submit your manuscripts at

http://www.hindawi.com

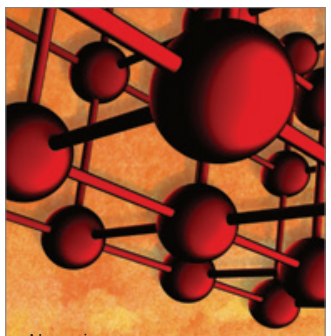

Materials Science and Engineering
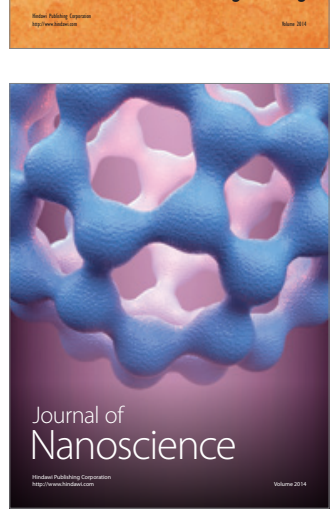
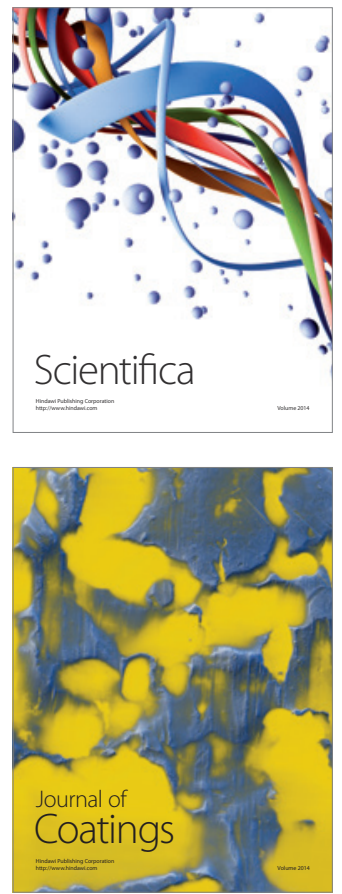
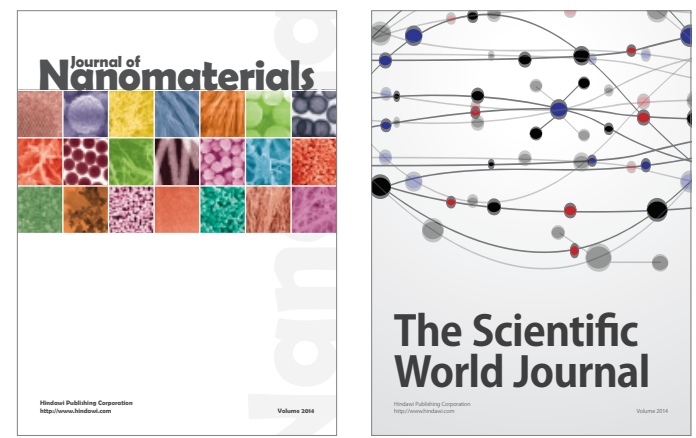

The Scientific World Journal
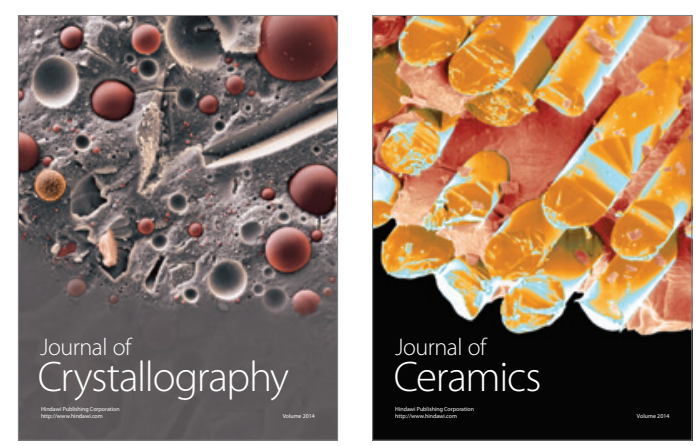
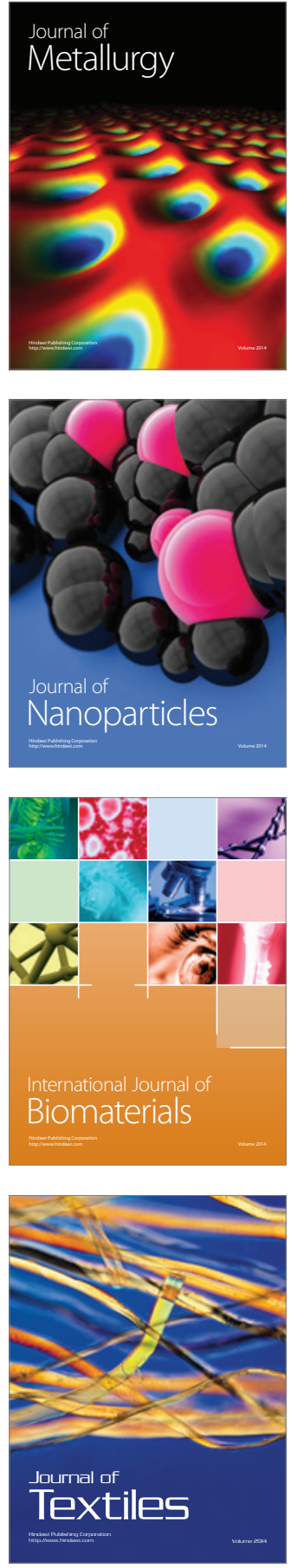